\title{
Counting Statistics of an Adiabatic Pump
}

\author{
Anton Andreev ${ }^{1}$, and Alex Kamenev ${ }^{2}$ \\ 1 Department of Physics, CB 390, University of Colorado, Boulder, CO 80303, USA. \\ 2 Department of Physics, Technion, Haifa 32000, Israel.
}

(November 1, 2018)

\begin{abstract}
We consider quantum statistics of charge transmitted through a mesoscopic device in the adiabatic pumping process. A general formula for the distribution function of the transmitted charge in terms of the time-dependent S-matrix is obtained. It is applied to a few simple examples of the pumping cycles. We identify coherent pumping strategies which lead to the binomial charge distribution and minimize the fluctuations of the pumped current. Conditions for the ideal noiseless quantized pump are discussed.

PACS numbers: 72.10.Bg, 73.22.-b, 05.45.+b
\end{abstract}

Adiabatic charge pumping has attracted considerable theoretical and experimental interest. It occurs when the Hamiltonian of the system is changed periodically with time. At the end of the pumping cycle a finite charge may be transmitted through the system. Such a charge transfer takes place even in the absence of any dc voltage applied to the system. The idea is originally due to Thouless [1], who showed that in certain one-dimensional systems the transmitted charge is quantized in the adiabatic limit. This mechanism has been proposed for making electric current standards [2].

Recently research has focussed on adiabatic pumping through mesoscopic devices [3 8]. Most of the work concentrated on the average pumping current and its mesoscopic fluctuations [3 6 . The general expression for the average transmitted charge was derived by Brouwer [4] in terms of the time-dependent $S$-matrix based on the formalism of Ref. [9]. The average charge transmitted during a cycle depends on the system's conductance and is not in general quantized 7.5.5. The absence of quantization is related to the fact that the true Thouless adiabatic conditions [1] can never be achieved for a system with infinite leads and hence a vanishing excitation gap. The possibility to create electron-hole pairs with arbitrarily small energy leads to dissipation and violates the exact quantization [5]. Taken together with the discrete nature of charge carriers this fact suggests the presence of thermal and shot noise in the adiabatic pumps. A better understanding of the noise characteristics of such devices is certainly necessary if the prospect of their use as current standards [8] is to be taken seriously.

In this Letter we address the problem of charge counting statistics in an adiabatic pump. We have greatly benefited from the extensive work on the current statistics of voltage-biased resistors by Levitov et. al. [10 12].

We consider a scatterer connected with the left and right leads, each having $n$ transverse channels 14. Such a scatterer is characterized by a $2 n \times 2 n$ unitary scattering matrix $S$, which can be written in the $n \times n$ block form

$$
S=\left(\begin{array}{cc}
r & t^{\prime} \\
t & r^{\prime}
\end{array}\right)
$$

Here $r\left(r^{\prime}\right)$ and $t\left(t^{\prime}\right)$ are left (right) reflection and transmission matrices correspondingly. We shall assume hereafter that the $S$-matrix is a periodic function of time $\tau$, with the period $\tau_{0}, S\left(\tau+\tau_{0}\right)=S(\tau)$. This timedependence is supposed to be adiabatic, i.e. $\tau_{0}$ is much greater than the Wigner delay time of the device. We restrict our attention to open systems, i.e. those with the dwell time shorter than the inverse mean level spacing in the device. Under these conditions one may neglect both the energy relaxation and the Coulomb blockade effects inside the device.

The transmission and reflection matrices in Eq. (11) can be simultaneously diagonalized by the block-diagonal unitary matrices $U(\tau)$ and $V(\tau)$ (see for example Ref. [16])

$$
S(\tau)=U(\tau) \tilde{S}(\tau) V^{\dagger}(\tau) .
$$

Here $\tilde{S}(\tau)$ is a matrix of the form Eq. (11) with real diagonal reflection and transmission blocks. The eigenvalues of the latter play the role of the instantaneous reflection and transmission amplitudes in the channels. The ambiguity in the definition of matrices $U(\tau)$ and $V(\tau)$ does not affect our final results.

In each cycle a number of electrons $Q$ may pass through the scattering region in a direction which depends on the detailed form of $S(\tau)$. The average charge transmitted in $N$ pumping cycles was recently given by Brouwer [4]

$$
\langle Q\rangle=\frac{1}{2 i} \int_{0}^{N \tau_{0}} \frac{d \tau}{2 \pi} \operatorname{Tr}\left\{\frac{\partial S}{\partial \tau} S^{\dagger} \sigma_{3}\right\},
$$

where charge $Q$ is measured in units of the electron charge. We have used the representation where the transmitted charge is written as half the sum of that through the left and through the right leads 15, thus $\sigma_{3}=\operatorname{diag}\{1,-1\}$ is a Pauli matrix with the $n \times n$ block structure. Both quantum and thermal noise lead to fluctuations in the transmitted charge $Q$. As a result the 
charge transmitted in one cycle can be described by a certain probability distribution.

Here we obtain the distribution function of transmitted charge for an adiabatic pump characterized by a periodic time-dependent $S$-matrix, $S(\tau)$. More precisely, we calculate the probability $P_{N}(Q)$ to transmit the charge $Q$ upon completion of $N$ pumping cycles. It is convenient to formulate the results in terms of the generating function $F_{N}(\lambda)$ of the moments of the transmitted charge defined as 10 .

$$
F_{N}(\lambda)=\left\langle e^{i Q \lambda}\right\rangle=\int d Q P_{N}(Q) e^{i Q \lambda} .
$$

For the $S$-matrix of the form Eq. (2) we obtain

$$
F_{N}(\lambda)=e^{i \hat{N} \lambda} \operatorname{det}\left[1+\tilde{S}_{\lambda}(\tau) \tilde{n}\left(\tau, \tau^{\prime}\right)\left(\tilde{S}_{-\lambda}^{\dagger}\left(\tau^{\prime}\right)-\tilde{S}_{\lambda}^{\dagger}\left(\tau^{\prime}\right)\right)\right],
$$

where

$$
\tilde{S}_{\lambda}(\tau) \equiv \exp \left\{-i \sigma_{3} \lambda / 4\right\} \tilde{S}(\tau) \exp \left\{i \sigma_{3} \lambda / 4\right\} .
$$

The matrix $\tilde{n}\left(\tau, \tau^{\prime}\right)$ is defined as

$$
\tilde{n}\left(\tau, \tau^{\prime}\right)=V^{\dagger}(\tau) \hat{n}\left(\tau-\tau^{\prime}\right) V\left(\tau^{\prime}\right) .
$$

Here the diagonal matrix $\hat{n}\left(\tau-\tau^{\prime}\right)$ is the time Fourier transform of $\hat{n}(\epsilon)=\operatorname{diag}\left\{n_{L}(\epsilon), n_{R}(\epsilon)\right\}$, where $n_{L(R)}(\epsilon)$ is the energy distribution function of the left (right) lead. The operator in the determinant in Eq. (5) should be understood as an operator in the time space as well as a matrix in the space of channels. Finally, the integer number $\hat{N}$ defined as

$$
\hat{N}=\frac{1}{2 i} \int_{0}^{N \tau_{0}} \frac{d \tau}{2 \pi} \operatorname{Tr}\left\{U^{\dagger} \frac{\partial U}{\partial \tau} \sigma_{3}-V^{\dagger} \frac{\partial V}{\partial \tau} \sigma_{3}\right\},
$$

is the contribution to the generating function arising from the chiral anomaly (see below). Equation (5) is the main result of this Letter. Before presenting the derivation, we shall illustrate its applications on a few examples.

Expanding $\ln F_{N}(\lambda)$ to the first power of $i \lambda$ one finds for the average transmitted charge, $\langle Q\rangle \equiv \sum_{Q} Q P_{N}(Q)$,

$$
\langle Q\rangle=\left.\int_{0}^{N \tau_{0}} \frac{d \tau}{4 \pi} \operatorname{Tr}\left\{\tilde{S}(\tau) \tilde{n}\left(\tau, \tau^{\prime}\right)\left[\sigma_{3}, \tilde{S}^{\dagger}\left(\tau^{\prime}\right)\right]\right\}\right|_{\tau^{\prime} \rightarrow \tau}+\hat{N} .
$$

In the absence of an external voltage $\tilde{n}\left(\tau, \tau^{\prime}\right) \rightarrow i /[2 \pi(\tau-$ $\left.\left.\tau^{\prime}+i \eta\right)\right]+i /(2 \pi) V^{\dagger}(\tau) \partial V(\tau) / \partial \tau$ for $\tau^{\prime} \rightarrow \tau$. Expanding $\tilde{S}^{\dagger}\left(\tau^{\prime}\right)$ to the first power in $\tau-\tau^{\prime}$ and using Eq. (8) one obtains Brouwer's result [4], Eq. (3]).

A particularly instructive example is given by a single channel case $(n=1)$ with the $S$-matrix depending on time as

$$
S(\tau)=e^{i \sigma_{3} \theta(\tau) / 2} \tilde{S} e^{i \sigma_{3} \theta(\tau) / 2},
$$

where $\theta\left(\tau+\tau_{0}\right)-\theta(\tau)=2 \pi$, and $\tilde{S}$ is time-independent. This form of the $S$-matrix is equivalent to the phase winding of the reflection amplitudes, $r \rightarrow r \exp \{i \theta(\tau)\}$ $\left(r^{\prime} \rightarrow r^{\prime} \exp \{-i \theta(\tau)\}\right)$. The contribution from the chiral anomaly in Eq. (8) coincides with the number of cycles, $\hat{N}=N$. We concentrate first on a particularly simple time-dependence, $\theta(\tau)=2 \pi \tau / \tau_{0}$. In this case the determinant in Eq. (5) may be easily calculated in the Fourier basis leading to

$$
\begin{aligned}
F_{N}(\lambda) & =\prod_{k}\left[1+n_{L}\left(\epsilon_{k+N}\right)\left(1-n_{R}\left(\epsilon_{k}\right)\right)\left(e^{i \lambda}-1\right)|t|^{2}\right. \\
& \left.+\left(1-n_{L}\left(\epsilon_{k+N}\right)\right) n_{R}\left(\epsilon_{k}\right)\left(e^{-i \lambda}-1\right)|t|^{2}\right] e^{i N \lambda}
\end{aligned}
$$

where $\epsilon_{k}=\pi(2 k+1) /\left(N \tau_{0}\right)$ are fermionic frequencies. At small temperature, $T \ll 1 / \tau_{0}, V$, this expression simplifies substantially, leading to

$$
F_{N}(\lambda)=\left(e^{i \lambda}|r|^{2}+|t|^{2}\right)^{N}\left(|r|^{2}+e^{-i \lambda}|t|^{2}\right)^{\frac{N \tau_{0} V}{2 \pi}},
$$

where $V$ is a voltage applied between the left and right leads, which is chosen to be such that $N \tau_{0} V /(2 \pi)$ is an integer [10]. In the absence of external voltage Eqs. (4) and (12) lead to the binomial distribution function of the charge transmitted through the adiabatic pump

$$
P_{N}(Q)=C_{N}^{Q}|r|^{2 Q}\left(1-|r|^{2}\right)^{N-Q} .
$$

Note that only the integer values of the transmitted charge have non-zero probability to be detected. The physical meaning of this expression is that each pumping cycle is associated with an attempt to transfer one electron. The success probability of such an attempt is given by the reflection probability, $|r|^{2}$, whereas the probability of failure is $1-|r|^{2}$. The above statistics should be compared with the case of the dc voltage applied across the scattering region (no pumping). This case may be obtained from Eq. (12) in the limit $N \rightarrow 0$, whereas $N \tau_{0} V /(2 \pi) \rightarrow \tilde{N}$ - integer. We immediately recover the familiar results 10

$$
P_{\tilde{N}}(Q)=C_{\tilde{N}}^{Q}|t|^{2 Q}\left(1-|t|^{2}\right)^{\tilde{N}-Q} .
$$

The distribution is also binomial, however the probability of success is given by the transmission probability, $|t|^{2}$. The second cumulant of the transmitted charge coincide for the adiabatic pump and applied dc voltage and is given by

$$
\left\langle\left\langle Q^{2}\right\rangle\right\rangle=|t|^{2}\left(1-|t|^{2}\right) N,
$$

leading to the maximal noise power in both cases at $|t|^{2}=|r|^{2}=1 / 2$.

The binomial distribution, Eq. (13) was derived above for the simplest time dependence of the form $r(\tau)=$ 
$r \exp \left\{2 \pi i \tau / \tau_{0}\right\}$. The same result may be shown to be valid for a more general class of pumping strategies, which we call the coherent pumping, following the terminology of Ref. [10,11:

$$
e^{i \theta(\tau)}=\frac{e^{2 \pi i \tau / \tau_{0}}-z}{1-z^{*} e^{2 \pi i \tau / \tau_{0}}}
$$

where $z$ is a complex number with $|z|<1$. Indeed, at zero temperature $n\left(\epsilon_{k}\right)=\theta(-2 k-1)$ has infinitely degenerate eigenvalues. One can therefore diagonalize the operator in Eq. (5), in the $z$-dependent basis which does not mix positive and negative frequencies. The value of the determinant does not depend on $z$ and one recovers the binomial distribution (13). Such coherent pumping strategy guarantees the minimal possible value of the noise in the transmitted charge. The general expression for the noise of the adiabatic pump may be derived by expanding $\ln F_{N}(\lambda)$, Eq. (5), to the second order in $i \lambda$ and is given by

$$
\left\langle\left\langle Q^{2}\right\rangle\right\rangle=\iint_{0}^{N \tau_{0}} \frac{d \tau d \tau^{\prime}}{\left(4 N \tau_{0}\right)^{2}} \frac{\operatorname{Tr}\left\{1-\left(S^{\dagger} \sigma_{3} S\right)_{\tau}\left(S^{\dagger} \sigma_{3} S\right)_{\tau^{\prime}}\right\}}{\sin ^{2}\left[\pi\left(\tau-\tau^{\prime}\right) /\left(N \tau_{0}\right)\right]} .
$$

Substituting the $S$-matrix of the form Eq. (10) and minimizing the charge fluctuations, $\left\langle\left\langle Q^{2}\right\rangle\right\rangle$, with respect to $e^{i \theta(t)}$ 13], one finds that the coherent pumping, Eqs. (10), (16), lead to the minimal possible noise. The value of this minimal noise is given by Eq. (15). Note also that the second moment, Eq. (17) (as well as the first one, Eq. (3), and the higher ones) may be expressed through the $S$-matrix only, rather than through auxiliary matrices defined in Eq. (2).

Next we consider a $2 \times 2$ scattering matrix with real reflection and transmission amplitudes given by $r=-r^{\prime}=$ $\cos \left(2 \pi \tau / \tau_{0}\right)$ and $t=t^{\prime}=\sin \left(2 \pi \tau / \tau_{0}\right)$ respectively. In this case $U(\tau)=V(\tau)=1$ leading to $\hat{N}=0$, and $\tilde{n}$ is the equilibrium distribution function. One can show that $F_{N}(\lambda)$ in Eq. (5) is an even function of $\lambda$, and is therefore real. As a result one may employ the method of Ref. [12] to compute the determinant of the operator in Eq. (5): one multiplies this operator by its Hermitian conjugate and takes the square root. The resulting operator may be written as $1+(1-\tilde{n}) \tilde{S}_{\lambda}^{\dagger} \tilde{S}_{-\lambda} \tilde{n}+\tilde{n} S_{-\lambda}^{\dagger} \tilde{S}_{\lambda}(1-\tilde{n})$. In the energy representation it has a finite number of the off-diagonal matrix elements and its determinant can be straightforwardly evaluated. This way one obtains

$$
F_{N}(\lambda)=\left(\frac{1+\cos \lambda}{2}\right)^{N}
$$

Therefore such pump is a realization of the "random walk motion" for charge. Indeed, there are three possible values of the transmitted charge in each cycle: $Q=0$ with the probability $1 / 2$, and $Q= \pm 1$ with the probability $1 / 4$ each.

We note that the logarithmic derivative $i V^{\dagger}(\tau) \partial V(\tau) / \partial \tau$ is analogous to the instantaneous matrix of "voltages" applied to the incoming channels. The integral of this quantity can be interpreted as the number of transmission attempts [10]. The different outcomes of such attempts lead to the noise of the pumping current. In general the probability distribution of the transmitted charge is not binomial. If the "voltage" matrix can not be diagonalized simultaneously with the reflection and transmission matrices then the distribution function of the transmitted charge does not factorize into binomial distributions of elementary transmission processes.

In contrast, the matrix $U(\tau)$ corresponds to the outgoing channels and enters the final expression (5) only through the chiral anomaly term (8) and therefore contributes to the average current but not to the noise. For example, the pumping cycle of the form $S(\tau)=U(\tau) \tilde{S}$ at zero temperature would produce a noiseless quantized pumping current.

We turn now to the derivation of Eq. (5). To this end we model the leads by a $2 n$-component vector of chiral incoming fermions $\left(\psi_{L}(x, \tau), \psi_{R}(x, \tau)\right)$ and $2 n$-component vector of chiral outgoing fermions $\left(\xi_{L}(x, \tau), \xi_{R}(x, \tau)\right)$. The action for e.g. left lead is written as

$$
S_{L}=\int_{\mathcal{C}} d \tau \int_{-\infty}^{0} d x \bar{\psi}_{L}\left(\partial_{t}+\hat{v}_{L} \partial_{x}\right) \psi_{L}+\bar{\xi}_{L}\left(\partial_{t}-\hat{v}_{L} \partial_{x}\right) \xi_{L}
$$

where $\hat{v}_{L}$ is a diagonal $n \times n$ matrix of the left lead channel velocities. In this expression the time integral runs along the Keldysh contour, $\mathcal{C}$, from $\tau=0$ to $\tau=N \tau_{0}$ and then back to $\tau=0$. The right lead is described by the similar action with the space integral running from $x=0$ to $x=+\infty$, and the velocity matrix $\hat{v}_{R}$. Finally the incoming and outgoing channels at $x=0$ are related by the time-dependent $S$-matrix operator

$$
\xi(0, \tau)=\hat{v}^{1 / 2} S(\tau) \hat{v}^{-1 / 2} \psi(0, \tau) .
$$

The current operator has a form $I=\left(I_{L}+I_{R}\right) / 2$, where

$$
I_{L}(\tau)=\left[\bar{\psi}_{L}\left(0^{-}, \tau\right) \hat{v}_{L} \psi_{L}\left(0^{-}, \tau\right)-\bar{\xi}_{L}\left(0^{-}, \tau\right) \hat{v}_{L} \xi_{L}\left(0^{-}, \tau\right)\right]
$$

The operator of the charge transmitted in $N$ cycles is given by $Q=\int_{0}^{N \tau_{0}} d \tau I(\tau)$. Finally, the generating function may be written as

$$
F_{N}(\lambda)=\int D[\psi, \xi] e^{-S_{L}-S_{R}+\frac{i}{2} \int_{\mathcal{C}} d \tau \hat{\lambda}(\tau) I(\tau)}
$$

where $\hat{\lambda}(\tau)$ is equal to $\lambda$ on the forward and $-\lambda$ on the backward part of the Keldysh contour. The fermion fields 
in this integral obey the boundary condition, Eq. 20. One has to specify the initial, $\tau=0$, density matrix, which implicitly defines the Green functions. We fix the occupation numbers in the incoming channels of the left and right leads to be $n_{L}(\epsilon)$ and $n_{R}(\epsilon)$ correspondingly, whereas the outgoing channels are supposed to be initially empty in accord with the scattering setup.

The subsequent calculations amount to the evaluation of the Gaussian integral in Eq. (22). To this end we first make the chiral gauge transformation of the fermionic fields: $\psi(x, \tau) \rightarrow V(\tau) \psi(x, \tau)$ and $\xi(x, \tau) \rightarrow U(t) \xi(x, \tau)$. As a result, the boundary condition for the new fermions contains the $\tilde{S}(\tau)$ matrix only and the action acquires an additional time-dependent (matrix) chemical potential term

$$
\delta S=\int_{\mathcal{C}} d \tau \int d x \bar{\psi}\left[V^{\dagger} \frac{\partial V}{\partial \tau}\right] \psi+\bar{\xi}\left[U^{\dagger} \frac{\partial U}{\partial \tau}\right] \xi .
$$

Such potential term results in the redefinition of the density matrix according to Eq. (7). Importantly, upon the chiral gauge transformation the expression for the current acquires an extra term $I \rightarrow$ $I+1 /(4 \pi i) \operatorname{Tr}\left\{\partial U^{\dagger}(\tau) \sigma_{3} U(\tau) / \partial \tau-\partial V^{\dagger}(\tau) \sigma_{3} V(\tau) / \partial \tau\right\}$ arising from the chiral anomaly [17].

Since the source field, $\hat{\lambda}(\tau)$, is a constant on the both branches of the Keldysh contour, one may eliminate the $\int \hat{\lambda} I$ term from the action by the time-independent gauge transformation [10], e.g. $\psi_{L} \rightarrow e^{i \theta\left(x-0^{-}\right) \lambda / 2} \psi_{L}$ on the forward branch of the contour and $\psi_{L} \rightarrow e^{-i \theta\left(x-0^{-}\right) \lambda / 2} \psi_{L}$ on the backward branch. Such transformation leads to the change in the phase of the forward scattering amplitude and can be taken into account by a redefinition of the $\tilde{S}$-matrix in the boundary condition, Eq. (20), $\tilde{S} \rightarrow \tilde{S}_{ \pm \lambda}$ on the forward (backward) branches, with $S_{\lambda}$ defined in Eq. (6).

The subsequent steps are straightforward. One integrates out all degrees of freedom except for those which reside directly at the scatterer, $x=0$. Using the boundary condition, Eq. (20), with $\tilde{S}_{ \pm \lambda}$-matrix, one eliminates the incoming degrees of freedom, $\psi(x=0, \tau)$. The remaining Gaussian integral over the outgoing fermions, $\xi(x=0, \tau)$, can be straightforwardly evaluated, resulting in the determinant written in Eq. (5). The remaining term, $\exp \{i \hat{N} \lambda\}$, is the contribution from the chiral anomaly, as explained above.

To conclude, we have derived a general expression for the counting statistics of the charge transmitted through a system described by a time-dependent $S$-matrix. The only limitations of our result are the requirements of adiabaticity and the absence of inelastic processes in the scattering region. The absolute minimum of the noise power may be achieved by the coherent pumping strategy, in which case the charge distribution is given by the product of binomial distributions. We point out the major role played by the chiral anomaly contribution to the average transmitted charge. Such anomalous term did not arise in the context of voltage-biased systems [10], but is extremely important for the adiabatic pumping setup.

It is our pleasure to acknowledge helpful discussions with I. Aleiner, Y. Avron, B. Spivak and L. Sadun. We appreciate the warm hospitality of the Norwegian Centre for Advanced Studies, where part of this work was performed. This work was partly supported through the grants BSF-9800338 and DMR-9984002. A. A. is an A. P. Sloan and Packard Fellow.

[1] D. J. Thouless, Phys. Rev. B 27, 6083 (1983).

[2] Q. Niu, Phys. Rev. Lett. 64, 1812 (1990).

[3] B. Spivak, F. Zhou, and M. T. Beal Monod, Phys. Rev. B 51, 13226 (1995); F. Zhou, B. Spivak, and B. L. Altshuler, Phys. Rev. Lett. 82, 608 (1999).

[4] P. W. Brouwer, Phys. Rev. B 58, R10135 (1998).

[5] T.A. Shutenko, I.L. Aleiner, and B.L. Altshuler, condmat/9911019.

[6] M. Switkes, C. M. Marcus, K. Campman and A. C. Gossard, Science, 283, 1905 (1999).

[7] I. L. Aleiner, and A. V. Andreev, Phys. Rev. Lett. 81, 1286 (1998).

[8] L.P. Kouwenhoven et. al. Phys. Rev. Lett. 67, 1626 (1991); M.H. Devoret, D. Esteve and C. Urbina, in Les Houches. Session LXI Mesoscopic Quantum Physics Eds. E. Akkermans, G. Montambaux, J.-L. Pichard and J. Zin-Justin (Elsevier, Amsterdam, 1995).

[9] M. Buttiker, H. Thomas, and A. Pretre, Z. Phys. B 94, 133 (1994).

[10] L. S. Levitov, and G. B. Lesovik, JETP Lett., 58, 230 (1993); L. S. Levitov, H. Lee, and G. B. Lesovik, J. Math. Phys. 37, 4845 (1996).

[11] D. A. Ivanov, H. W. Lee, and L. S. Levitov, Phys. Rev. B 56, 6839 (1997).

[12] D. A. Ivanov, and L. S. Levitov, JETP Lett. 58, 461 (1993).

[13] S. Korshunov, JETP Lett., 45, 434 (1987).

[14] One can always add some ficticious non-conducting channels to one of the leads to make the number of channels in each lead equal.

[15] To have the average charge passing in and out the system equal, the $S$-matrix should satisfy $\int_{0}^{\tau_{0}} d \tau \operatorname{Tr}\left\{S^{\dagger} \partial S / \partial \tau\right\}=0$.

[16] A. D. Stone, P. Mello, and Muttalib, in Mesoscopic Phenomena in Solids B. L. Altshuler, P. A. Lee, R. A. Wibb editors, Elsevier, 1991.

[17] J. Zinn-Justin Quantum Field Theory and Critical Phenomena, Clarendon Press, Oxford, 1989. 